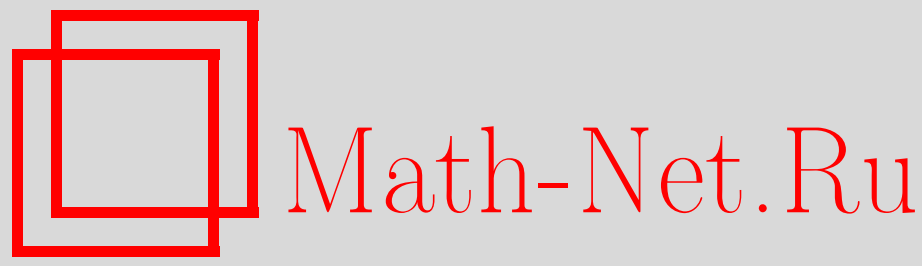

Б. Г. Драгович, Дзета-нелокальные скалярные поля, TMФ, 2008, том 157, номер 3, 364-372

DOI: https://doi.org/10.4213/tmf6285

Использование Общероссийского математического портала Math-Net.Ru подразумевает, что вы прочитали и согласны с пользовательским соглашением http://www . mathnet.ru/rus/agreement

Параметры загрузки:

IP: 18.208 .226 .222

26 апреля 2023 г., 11:29:01

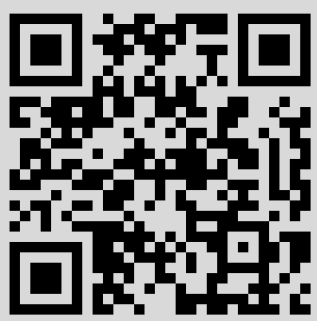




\title{
ДЗЕТА-НЕЛОКАЛЬНЫЕ СКАЛЯРНЫЕ ПОЛЯ
}

\begin{abstract}
Рассмотрены некоторые нелокальные и неполиномиальные модели скалярного поля, возникающие в теории $p$-адических струн. Бесконечное число пространственно-временных производных определяется операторнозначной дзетафункцией Римана через даламбертиан $\square$ в качестве ее аргумента. Соответствующие лагранжианы $L$ строятся исходя из точного лагранжиана $\mathcal{L}_{p}$ для эффективного поля $p$-адической тахионной струны, который обобщается посредством замены $p$ на произвольное натуральное число $n$ и суммированием $\mathcal{L}_{n}$ по всем $n$. Получен ряд основных классических свойств таких полей. В частности, изучены некоторые решения уравнений движения и их тахионные спектры. Теория поля с динамикой дзета-функции Римана интересна также и сама по себе.
\end{abstract}

Ключевые слова: нелокальная теория поля, $p$-адическая теория струн, дзета-функция Римана.

Посвящается Василию Сергеевичу Владимирову в связи с 85-летием

\section{1. ВВЕДЕНИЕ}

Прошло уже два десятилетия со времени выхода первой публикации о $p$-адической струне [1]. K настоящему моменту разнообразные $p$-адические структуры наблюдались не только в теории струн, но и во многих других моделях современной математической физики (обзор развития теории струн см., например, в работах [2], [3]). Одно из наиболее значительных достижений теории $p$-адических струн состоит в эффективном полевом описании открытых скалярных р-адических струн [4], [5]. Эффективный тахионный лагранжиан очень прост и точен. Он описывает не только четырехточечные, но и все высшие амплитуды рассеяния на древесном уровне.

Теория $p$-адических струн получила значительное развитие, когда было показано [6], что она описывает тахионную конденсацию и бранные соотношения спуска проще, чем теория обычных бозонных струн. Вслед за этим были исследованы многие аспекты динамики $p$-адических струн в сравнении с динамикой обычных струн (см., например, [7]-[10] и приведенную там библиографию). Некоммутативная деформация мирового листа $p$-адической струны с постоянным $B$-полем исследовалась

*Institute of Physics, Belgrade, Serbia. E-mail: dragovich@phy.bg.ac.yu 
в работах [11] (см. также [12]). Значительный интерес представляет систематическое математическое исследование пространственно однородных решений соответствующих нелинейных дифференциальных уравнений движения (см. [9], [13]-[15] и приведенную там библиографию). Исследовались также некоторые возможные космологические следствия из теории р-адических струн [16]-[20]. В предположении, что теории $p$-адических струн дают решеточную дискретизацию мирового листа обычных струн [21], удалось воспроизвести некоторые нетривиальные свойства теории обыкновенных струн исходя из $p$-адического эффективного действия. Более того, были установлены многочисленные сходства и аналогии между $p$-адическими и обыкновенными струнами.

С помощью адельного подхода к струнным амплитудам рассеяния можно установить связь между р-адическими явлениями и их обычными аналогами [2], [3]. Более того, этот подход позволяет исключить параметр, задаваемый простым числом $p$, который содержится в $p$-адических амплитудах, а также исправить ситуацию, связанную с проблемой $p$-адического нарушения причинности. Было также сформулировано адельное обобщение квантовой механики и найдена связь между адельным вакуумным состоянием гармонического осциллятора и дзета-функцией Римана [22]. Интересный подход к обоснованию теории поля и космологии, основанный на использовании дзета-функции Римана, был предложен в работе [23]. Заметим, что и p-адический, и обычный секторы четырехточечных адельных струнных амплитуд содержат дзета-функцию Римана (см., например, [2], [3], [24]).

Основная цель настоящей работы - получить соответствующий эффективный лагранжиан для адельной скалярной струны. Поэтому сначала мы исследуем как можно вывести лагранжиан, связанный с p-адическим сектором адельной струны. Исходя из точного лагранжиана для эффективного поля $p$-адической тахионной струны, переходя от простого числа $p$ к произвольному натуральному числу $n$ и выполняя различные суммирования таких лагранжианов по всем $n$, получаем некоторые скалярные теории поля с операторнозначной дзета-функцией Римана. Дзета-функцию Римана, появляющуюся на классическом уровне, можно рассматривать как ее аналог в квантовой амплитуде рассеяния. Будет показано, что эта дзета-функция "управляет" пространственно-временной нелокальностью. Далее мы построим и исследуем некоторые классические полевые модели, с помощью которых можно будет описать некоторые свойства адельной открытой скалярной струны.

\section{2. МОДЕЛИРОВАНИЕ НЕКОТОРЫХ ДЗЕТА-НЕЛОКАЛЬНЫХ СКАЛЯРЫХ ПОЛЕЙ}

На древесном уровне точный лагранжиан эффективного скалярного поля $\varphi$, описывающего тахион в открытой $p$-адической струне, имеет вид

$$
\mathcal{L}_{p}=\frac{m_{p}^{D}}{g_{p}^{2}} \frac{p^{2}}{p-1}\left[-\frac{1}{2} \varphi p^{-\square /\left(2 m_{p}^{2}\right)} \varphi+\frac{1}{p+1} \varphi^{p+1}\right],
$$

где $p$ - любое простое число, а $\square=-\partial_{t}^{2}+\nabla^{2}-D$-мерный даламбертиан; мы используем метрику с сигнатурой $(-,+, \ldots,+)$. Бесконечное число пространственно-вре- 
менных производных возникает из разложения

$$
p^{-\square /\left(2 m_{p}^{2}\right)}=\exp \left[-\frac{1}{2 m_{p}^{2}} \ln p \square\right]=\sum_{k=0}^{\infty}\left(-\frac{\ln p}{2 m_{p}^{2}}\right)^{k} \frac{1}{k !} \square^{k} .
$$

Уравнения движения для лагранжиана (1) имеют вид

$$
p^{-\square /\left(2 m_{p}^{2}\right)} \varphi=\varphi^{p}
$$

Уравнение (2) исследовалось многими авторами (см., например, [9], [13]-[15] и приведенную там библиографию).

Стоит отметить, что простое число $p$ в (1) и (2) можно заменить любым натуральным числом $n \geqslant 2$ так, что выражения по-прежнему будут иметь смысл. Более того, при $p=1+\varepsilon \rightarrow 1$ выражение для лагранжиана (1) имеет предел

$$
\mathcal{L}=\frac{m^{D}}{g^{2}}\left[\frac{1}{2} \varphi \frac{\square}{m^{2}} \varphi+\frac{\varphi^{2}}{2}\left(\ln \varphi^{2}-1\right)\right],
$$

который связан с обыкновенной бозонной струной в струнной теории поля с границей [25].

Введем модель, включающую в себя все струнные лагранжианы (1), в которых $p$ заменено на $n \in \mathbb{N}$. Для этого возьмем сумму всех лагранжианов $\mathcal{L}_{n}$ в виде

$$
L=\sum_{n=1}^{\infty} C_{n} \mathcal{L}_{n}=\sum_{n=1}^{\infty} C_{n} \frac{m_{n}^{D}}{g_{n}^{2}} \frac{n^{2}}{n-1}\left[-\frac{1}{2} \phi n^{-\square /\left(2 m_{n}^{2}\right)} \phi+\frac{1}{n+1} \phi^{n+1}\right]
$$

где явная реализация зависит от конкретного выбора коэффициентов $C_{n}$, масс $m_{n}$ и постоянных взаимодействия $g_{n}$. Чтобы избежать проблемы с расходимостью $1 /(n-1)$ при $n=1$, следует учесть, что $C_{n} m_{n}^{D} / g_{n}^{2}$ пропорционально $n-1$. В данной работе мы рассмотрим случай, когда коэффициенты $C_{n}$ пропорциональны $n-1$, а массы $m_{n}$ и константы взаимодействия $g_{n}$ не зависят от $n$, т.е. $m_{n}=m, g_{n}=g$. Поскольку рассматривается подход к эффективному лагранжиану адельной струны, имеет смысл выбрать массу и константу взаимодействия, не зависящие от конкретных $p$ или $n$. Пусть адельный физический объект имеет фиксированные параметры с рациональными значениями. Чтобы подчеркнуть, что лагранжиан (4) описывает новое поле, отличное от частного $p$-адического, введем обозначение $\phi$ вместо $\varphi$. При $n=1$ оба слагаемых в (4) равны с точностью до знака, но мы оставим их, поскольку они обеспечивают подходящий вид полного лагранжиана $L$.

Рассмотрим простой случай

$$
C_{n}=\frac{n-1}{n^{2+h}},
$$

где $h$ - вещественное число. Соответствующий лагранжиан имеет вид

$$
L_{h}=\frac{m^{D}}{g^{2}}\left[-\frac{1}{2} \phi \sum_{n=1}^{\infty} n^{-\square /\left(2 m^{2}\right)-h} \phi+\sum_{n=1}^{\infty} \frac{n^{-h}}{n+1} \phi^{n+1}\right]
$$

и зависит от параметра $h$. 
Согласно формуле Эйлера можно записать

$$
\sum_{n=1}^{\infty} n^{-\square /\left(2 m^{2}\right)-h}=\prod_{p} \frac{1}{1-p^{-\square /\left(2 m^{2}\right)-h}} .
$$

Напомним, что стандартное определение дзета-функции Римана имеет вид

$$
\zeta(s)=\sum_{n=1}^{\infty} \frac{1}{n^{s}}=\prod_{p} \frac{1}{1-p^{-s}}, \quad s=\sigma+i \tau, \quad \sigma>1 .
$$

Это выражение допускает аналитическое продолжение на всю плоскость комплексных $s$, за исключением точки $s=1$, где оно имеет простой полюс с вычетом 1 . Пользуясь определением (7), можно переписать (6) в виде

$$
L_{h}=\frac{m^{D}}{g^{2}}\left[-\frac{1}{2} \phi \zeta\left(\frac{\square}{2 m^{2}}+h\right) \phi+\sum_{n=1}^{\infty} \frac{n^{-h}}{n+1} \phi^{n+1}\right] .
$$

Здесь $\zeta\left(\square /\left(2 m^{2}\right)+h\right)$ действует как псевдодифференциальный оператор

$$
\zeta\left(\frac{\square}{2 m^{2}}+h\right) \phi(x)=\frac{1}{(2 \pi)^{D}} \int e^{i x k} \zeta\left(-\frac{k^{2}}{2 m^{2}}+h\right) \tilde{\phi}(k) d k,
$$

где $\tilde{\phi}(k)=\int e^{-i k x} \phi(x) d x-$ преобразование Фурье функции $\phi(x)$. Лагранжиан $L_{0}$ с ограничением на импульсы $-k^{2}=k_{0}^{2}-\vec{k}^{2}>(2-2 h) m^{2}$ и поле $|\phi|<1$ исследовались в работе [26]. Ниже мы рассмотрим лагранжиан (8) с аналитическим продолжением дзета-функции и степенным рядом $\sum n^{-h} \phi^{n+1} /(n+1)$, т.е.

$$
L_{h}=\frac{m^{D}}{g^{2}}\left[-\frac{1}{2} \phi \zeta\left(\frac{\square}{2 m^{2}}+h\right) \phi+\mathcal{A C} \sum_{n=1}^{\infty} \frac{n^{-h}}{n+1} \phi^{n+1}\right],
$$

где $\mathcal{A C}$ означает аналитическое продолжение.

Нелокальная динамика этого поля $\phi$ содержится в псевдодифференциальном виде дзета-функции Римана. Когда в аргументе дзета-функции Римана присутствует даламбертиан, мы будем говорить, что имеется дзета-нелокальность. Соответственно указанное поле $\phi$ является дзета-нелокальным скалярным полем.

Потенциал указанного дзета-скалярного поля (10) равен $-L_{h}$ при $\square=0$, т.е.

$$
V_{h}(\phi)=\frac{m^{D}}{g^{2}}\left(\frac{\phi^{2}}{2} \zeta(h)-\mathcal{A C} \sum_{n=1}^{\infty} \frac{n^{-h}}{n+1} \phi^{n+1}\right),
$$

где $h \neq 1$, поскольку $\zeta(1)=\infty$. Слагаемое с дзета-функцией обращается в нуль при $h=-2,-4,-6, \ldots$.

Уравнения движения в дифференциальном и интегральном виде записываются в виде

$$
\begin{aligned}
\zeta\left(\frac{\square}{2 m^{2}}+h\right) \phi & =\mathcal{A C} \sum_{n=1}^{\infty} n^{-h} \phi^{n}, \\
\frac{1}{(2 \pi)^{D}} \int_{\mathbb{R}^{D}} e^{i x k} \zeta\left(-\frac{k^{2}}{2 m^{2}}+h\right) \tilde{\phi}(k) d k & =\mathcal{A C} \sum_{n=1}^{\infty} n^{-h} \phi^{n} .
\end{aligned}
$$


Очевидно, что $\phi=0$ является тривиальным решением для любого вещественного $h$. Существование других тривиальных решений зависит от параметра $h$. При $h>1$ имеется другое тривиальное решение $\phi=1$.

В приближении слабого поля (т.е. при $|\phi(x)| \ll 1)$ выражение (13) принимает вид

$$
\int_{\mathbb{R}^{D}} e^{i k x}\left[\zeta\left(-\frac{k^{2}}{2 m^{2}}+h\right)-1\right] \tilde{\phi}(k) d k=0 .
$$

Уравнение (14) имеет решение $\tilde{\phi}(k) \neq 0$, если выполнено условие

$$
\zeta\left(-\frac{k^{2}}{2 m^{2}}+h\right)=1 .
$$

С учетом обычного соотношения $k^{2}=-k_{0}^{2}+\vec{k}^{2}=-M^{2}$ уравнение (15) переписывается в виде

$$
\zeta\left(\frac{M^{2}}{2 m^{2}}+h\right)=1
$$

и определяет спектр масс $M^{2}=\mu_{h} m^{2}$, где набор значений спектральной функции $\mu_{h}$ зависит от $h$.

Уравнение (16) дает бесконечное множество решений с тахионной массой. А именно, функция $\zeta(s)$ непрерывна для вещественных $s \neq 1$ и меняет знак при переходе через нули $s=-2 n, n \in \mathbb{N}$. Согласно соотношению $\zeta(1-2 n)=-B_{2 n} /(2 n)$ и в соответствии со значениями чисел Бернулли $\left(B_{0}=1, B_{1}=-1 / 2, B_{2}=1 / 6, B_{4}=-1 / 30\right.$, $B_{6}=1 / 42, B_{8}=-1 / 30, B_{10}=5 / 66, B_{12}=-691 / 2730, B_{14}=7 / 6, B_{16}=-3617 / 510$, $\left.B_{18}=43867 / 798, \ldots\right)$ получается, что $|\zeta(1-2 n)|=\left|B_{2 n} /(2 n)\right|>1$ тогда и только тогда, когда $n \geqslant 9$. С учетом областей, где $\zeta(1-2 n)>0$, мы заключаем, что уравнение $\zeta(s)=1$ имеет два решения при $-20-4 j<s<-18-4 j$ для каждого $j=0,1,2, \ldots$. Соответственно для любого $h \in \mathbb{Z}$ получаем бесконечно много тахионных масс

$$
M^{2}=-\left(40+8 j+2 h-a_{j}\right) m^{2} \quad \text { и } \quad M^{2}=-\left(36+8 j+2 h+b_{j}\right) m^{2},
$$

где $a_{j} \ll 1, b_{j} \ll 1$ и $j=0,1,2, \ldots$.

\section{3. РЕЗУЛЬТАТЫ ДЛЯ НЕКОТОРЫХ ЗНАЧЕНИЙ $h$}

Среди бесконечно большого числа возможных значений $h$ в (5) рассмотрим пять $(h=0, \pm 1, \pm 2)$, которые представляют наибольший интерес. Случай $h=-2$ соответствует простейшему виду коэффициентов $C_{n}$, которые содержат $n-1$. В случае $h=-1$ коэффициенты $C_{n}=(n-1) / n \rightarrow 1$ при больших $n$ и лагранжианы $\mathcal{L}_{n}$ учитываются почти единообразно. В случае $h=0$ коэффициенты $C_{n}=(n-1) / n^{2}$ обратны соответствующим значениям из $\mathcal{L}_{n}$, что сильно упрощает полученные выражения. Анализ случаев $h=1$ и $h=2$ позволяет получить более глубокое представление о поведении лагранжиана $L_{h}$ вблизи $h=0$. 
3.1. Случай $h=-2$. Лагранжиан (10), соответствующий потенциал и уравнение движения имеют вид

$$
\begin{gathered}
L_{-2}=\frac{m^{D}}{g^{2}}\left[-\frac{1}{2} \phi \zeta\left(\frac{\square}{2 m^{2}}-2\right) \phi+\frac{2 \phi^{2}-\phi}{(1-\phi)^{2}}-\frac{1}{2} \ln (1-\phi)^{2}\right], \\
V_{-2}(\phi)=\frac{m^{D}}{g^{2}}\left[\frac{\phi-2 \phi^{2}}{(1-\phi)^{2}}+\frac{1}{2} \ln (1-\phi)^{2}\right], \\
\zeta\left(\frac{\square}{2 m^{2}}-2\right) \phi=\frac{1}{(2 \pi)^{D}} \int_{\mathbb{R}^{D}} e^{i x k} \zeta\left(-\frac{k^{2}}{2 m^{2}}-2\right) \tilde{\phi}(k) d k=\frac{\phi(\phi+1)}{(1-\phi)^{3}} .
\end{gathered}
$$

Потенциал $V_{-2}(\phi)$ имеет один локальный минимум $V_{-2}(-1) \approx-0.057 m^{D} / g^{2}$ и один локальный максимум $V_{-2}(0)=0$. Он сингулярен при $\phi=1$ (т.е. $\lim _{\phi \rightarrow 1} V_{-2}(\phi)=$ $-\infty)$ и, кроме того, $\lim _{\phi \rightarrow \pm \infty} V_{-2}(\phi)=+\infty$. Уравнение движения (20) имеет два тривиальных решения: $\phi(x)=0$ и $\phi(x)=-1$. Решение $\phi(x)=-1$ можно также получить, если взять $\tilde{\phi}(k)=-\delta(k)(2 \pi)^{D}$ и учесть, что $\zeta(-2)=0$ в $(20)$.

3.2. Случай $h=-1$. Соответствующий лагранжиан, потенциал и уравнение движения имеют вид

$$
\begin{gathered}
L_{-1}=\frac{m^{D}}{g^{2}}\left[-\frac{1}{2} \phi \zeta\left(\frac{\square}{2 m^{2}}-1\right) \phi+\frac{\phi}{1-\phi}+\frac{1}{2} \ln (1-\phi)^{2}\right], \\
V_{-1}(\phi)=\frac{m^{D}}{g^{2}}\left[\frac{\zeta(-1)}{2} \phi^{2}-\frac{\phi}{1-\phi}-\frac{1}{2} \ln (1-\phi)^{2}\right], \\
\zeta\left(\frac{\square}{2 m^{2}}-1\right) \phi=\frac{1}{(2 \pi)^{D}} \int_{\mathbb{R}^{D}} e^{i x k} \zeta\left(-\frac{k^{2}}{2 m^{2}}-1\right) \tilde{\phi}(k) d k=\frac{\phi}{(1-\phi)^{2}},
\end{gathered}
$$

где $\zeta(-1)=-1 / 12$. Потенциал (22) обладает следующими свойствами: он имеет локальный максимум $V_{-1}(0)=0, \lim _{\phi \rightarrow 1-0} V_{-1}(\phi)=-\infty, \lim _{\phi \rightarrow 1+0} V_{-1}(\phi)=+\infty$, $\lim _{\phi \rightarrow \pm \infty} V_{-1}(\phi)=-\infty$, для него отсутствует устойчивый вакуум. Уравнение движения (23) имеет постоянное тривиальное решение только при $\phi(x)=0$.

3.3. Случай $h=0$. Соответствующий лагранжиан имеет вид

$$
L_{0}=-\frac{m^{D}}{g^{2}}\left[\frac{1}{2} \phi \zeta\left(\frac{\square}{2 m^{2}}\right) \phi+\phi+\frac{1}{2} \ln (1-\phi)^{2}\right] .
$$

Потенциал принимает вид

$$
V_{0}(\phi)=\frac{m^{D}}{g^{2}}\left[\frac{\zeta(0)}{2} \phi^{2}+\phi+\frac{1}{2} \ln (1-\phi)^{2}\right],
$$

где $\zeta(0)=-1 / 2$. Он имеет два локальных максимума: $V_{0}(0)=0$ и $V_{0}(3) \approx$ $1.443 m^{D} / g^{2}$, нет устойчивых точек и $\lim _{\phi \rightarrow 1} V_{0}(\phi)=-\infty, \lim _{\phi \rightarrow \pm \infty} V_{0}(\phi)=-\infty$. Уравнение движения имеет вид

$$
\zeta\left(\frac{\square}{2 m^{2}}\right) \phi=\frac{1}{(2 \pi)^{D}} \int_{\mathbb{R}^{D}} e^{i x k} \zeta\left(-\frac{k^{2}}{2 m^{2}}\right) \tilde{\phi}(k) d k=\frac{\phi}{1-\phi} .
$$


Оно имеет два решения: $\phi=0$ и $\phi=3$. Решение $\phi=0$ очевидно, а решение $\phi=3$ следует из разложения Тейлора дзета-функции Римана

$$
\zeta\left(\frac{\square}{2 m^{2}}\right)=\zeta(0)+\sum_{n \geqslant 1} \frac{\zeta^{(n)}(0)}{n !}\left(\frac{\square}{2 m^{2}}\right)^{n}
$$

при $\tilde{\phi}(k)=(2 \pi)^{D} 3 \delta(k)$.

3.4. Случай $h=1$. Аналогично предыдущему случаю имеем

$$
\begin{gathered}
L_{1}=\frac{m^{D}}{g^{2}}\left[-\frac{1}{2} \phi \zeta\left(\frac{\square}{2 m^{2}}+1\right) \phi+\phi+\frac{1-\phi}{2} \ln (1-\phi)^{2}\right], \\
V_{1}(\phi)=\frac{m^{D}}{g^{2}}\left[\frac{\zeta(1)}{2} \phi^{2}-\phi-\frac{1-\phi}{2} \ln (1-\phi)^{2}\right], \\
\frac{1}{(2 \pi)^{D}} \int_{\mathbb{R}^{D}} e^{i x k} \zeta\left(-\frac{k^{2}}{2 m^{2}}+1\right) \tilde{\phi}(k) d k=-\frac{1}{2} \ln (1-\phi)^{2},
\end{gathered}
$$

где $V_{1}(\phi)=\infty$, поскольку $\zeta(1)=\infty$.

3.5. Случай $h=2$. В этом случае имеем

$$
\begin{gathered}
L_{2}=\frac{m^{D}}{g^{2}}\left[-\frac{1}{2} \phi \zeta\left(\frac{\square}{2 m^{2}}+2\right) \phi-\frac{1-\phi}{2} \ln (1-\phi)^{2}-\phi-\phi \int_{0}^{\phi} \frac{\ln (1-w)^{2}}{2 w} d w\right], \\
V_{2}(\phi)=\frac{m^{D}}{g^{2}}\left[\frac{\zeta(2)}{2} \phi^{2}+\frac{1-\phi}{2} \ln (1-\phi)^{2}+\phi+\phi \int_{0}^{\phi} \frac{\ln (1-w)^{2}}{2 w} d w\right], \\
\frac{1}{(2 \pi)^{D}} \int_{\mathbb{R}^{D}} e^{i x k} \zeta\left(-\frac{k^{2}}{2 m^{2}}+2\right) \tilde{\phi}(k) d k=-\int_{0}^{\phi} \frac{\ln (1-w)^{2}}{2 w} d w .
\end{gathered}
$$

Поскольку выполнено равенство

$$
-\int_{0}^{1} \frac{\ln (1-w)}{w} d w=\sum_{n=1}^{\infty} \frac{1}{n^{2}}=\zeta(2),
$$

уравнение (33) имеет тривиальное решение $\phi=1$.

\section{4. ЗАКЛЮЧИТЕЛЬНЫЕ ЗАМЕЧАНИЯ}

При построении эффективной теории поля адельной открытой скалярной струны мы вывели несколько лагранжианов, которые содержат все соответствующие $n$-адические лагранжианы $(n \in \mathbb{N})$. В результате получено, что бесконечное число пространственно-временных производных и соответствующая нелокальность "управляются" дзета-функцией Римана. Потенциалы неполиномиальны, спектр масс тахиона определяется некоторыми уравнениями, p-адические лагранжианы легко восстановить из дзета-лагранжиана, используя для их построения обратную процедуру. 
В данной работе описаны некоторые основные классические свойства введенного дзета-скалярного поля. Остается еще много вопросов, которые необходимо исследовать в классическом случае. Один из них - систематическое изучение уравнений движения и нетривиальных решений. В квантовом случае было бы полезно исследовать амплитуды рассеяния и провести сравнение с адельной струной.

Используя приведенную выше процедуру, имеет смысл рассматривать конструкцию лагранжиана для открыто-замкнутой дзета-струны исходя из $p$-адического лагранжиана [2]. Заметим, что эффективный лагранжиан для открыто-замкнутых $p$-адических струн можно также использовать для анализа тахионной конденсации [27].

Благодарности. Данная работа была выполнена при частичной поддержке со стороны Министерства науки Сербии, контракт № 144032D. Автор благодарит И. Я. Арефьеву, В. С. Владимирова и И. В. Воловича за полезные обсуждения, Д. Гошала за ряд замечаний и П.Г.О. Фрейнда, предложившего автору рассмотреть адельный подход. Данная работа выполнена во время пребывания автора в Математическом институте им. В. А. Стеклова в Москве.

\section{Список литературы}

[1] И. В. Волович, ТМФ, 71:3 (1987), 337-340; I. V. Volovich, Class. Quantum Grav., 4:4 (1987), L83-L87.

[2] L. Brekke, P. G. O. Freund, Phys. Rep., 233:1 (1993), 1-66.

[3] В.С. Владимиров, И. В. Волович, Е. И. Зеленов, p-Адический анализ и математическая физика, Наука, М., 1994.

[4] L. Brekke, P. G. O. Freund, M. Olson, E. Witten, Nucl. Phys. B, 302:3 (1988), 365-402.

[5] P. H. Frampton, Y. Okada, Phys. Rev. D, 37:10 (1988), 3077-3079.

[6] D. Ghoshal, A. Sen, Nucl. Phys. B, 584:1-2 (2000), 300-312; arXiv: hep-th/0003278.

[7] J.A. Minahan, JHEP, 03 (2001), 028; arXiv: hep-th/0102071.

[8] A. Sen, JHEP, 10 (2002), 003; arXiv: hep-th/0207105.

[9] N. Moeller, B. Zwiebach, JHEP, 10 (2002), 034; arXiv: hep-th/0207107.

[10] I. Ya. Aref'eva, L. V. Joukovskaya, A. S. Koshelev, JHEP, 09 (2003), 012; arXiv: hep-th/0301137.

[11] D. Ghoshal, T. Kawano, Nucl. Phys. B, 710:3 (2005), 577-598; arXiv: hep-th/0409311; P. Grange, Phys. Lett. B, 616:1-2 (2005), 135-140; arXiv: hep-th/0409305.

[12] B. Dragovich, I. V. Volovich, "p-Adic strings and noncommutativity", Noncommutative Structures in Mathematics and Physics, NATO Sci. Ser. II, Math. Phys. Chem., 22, eds. S. Duplij, J. Wess, Kluwer, Dordrecht, 2001, 391-399; D. Ghoshal, JHEP, 09 (2004), 041; arXiv: hep-th/0406259.

[13] В.С. Владимиров, Я.И. Волович, ТМФ, 138:3 (2004), 355-368; arXiv: math-ph/0306018.

[14] В. С. Владимиров, ТМФ, 149:3 (2006), 354-367; arXiv: 0705.4600.

[15] N. Barnaby, N. Kamran, Dynamics with infinitely many derivatives: the initial value problem, arXiv: 0709.3968v1.

[16] I. Ya. Aref'eva, "Nonlocal string tachyon as a model for cosmological dark energy", $p$-Adic Mathematical Physics, AIP Conf. Proc., 826, AIP, New York, 2006, 301-311; arXiv: astro-ph/0410443.

[17] И. Я. Арефьева, И. В. Волович, ТМФ, 155:1 (2008), 3-12; arXiv: hep-th/0612098.

[18] N. Barnaby, T. Biswas, J. M. Cline, JHEP, 04 (2007), 056; arXiv: hep-th/0612230. 
[19] I. Ya. Aref'eva, L. V. Joukovskaya, S. Yu. Vernov, JHEP, 07 (2007), 087; arXiv: hep-th/0701184.

[20] G. Calcagni, M. Montobbio, G. Nardelli, Phys. Rev. D, 76:12 (2007), 126001; arXiv: 0705.3043.

[21] D. Ghoshal, Phys. Rev. Lett., 97:15 (2006), 151601.

[22] Б. Г. Драгович, ТМФ, 101:3 (1994), 349-359; Internat. J. Modern Phys. A, 10:16 (1995), 2349-2365; arXiv: hep-th/0404160.

[23] I. Ya. Aref'eva, I. V. Volovich, Int. J. Geom. Methods Mod. Phys., 4:5 (2007), 881-895; arXiv: hep-th/0701284.

[24] I. Ya. Aref'eva, B. G. Dragovich, I. V. Volovich, Phys. Lett. B, 209:4 (1988), 445-450.

[25] A. Gerasimov, S. Shatashvili, JHEP, 10 (2000), 034; arXiv: hep-th/0009103.

[26] B. Dragovich, Zeta strings, arXiv: hep-th/0703008.

[27] N. Moeller, M. Schnabl, JHEP, 01 (2004), 011; arXiv: hep-th/0304213.

Поступила в редакцию 25.04.2008 